\title{
Effect of Blade Exit Shape on Performance and Vibration of a Double Volute Centrifugal Pump
}

\author{
Atia E. Khalifa
}

\begin{abstract}
The fluid-structure interaction phenomenon is the main cause of flow-induced vibrations at the blade passing frequency in large and high pressure centrifugal pumps. One way to reduce the effects of this interaction and pump vibration is to increase the effective gap by cutting the blade exit of the impeller. However, this cut of the impeller blade will affect the pump head and the flow pattern inside the pump volute. A single stage of a double volute centrifugal pump is used to investigate the effect of increasing the effective gap, by cutting the impeller blade exit with different shapes, on pump performance and vibration. Different cut shapes are tested, namely $\mathrm{V}$-shaped cut, C-shaped cut, and straight cut. Results showed the minimum pressure fluctuations inside the pump agree with the minimum pump vibration at the best efficiency point of the pump. Operating at off-design conditions increases the pump vibration. The cut at the blade exit increases the effective gap between the impeller and volute tongues and allows the dissipation of pressure pulse energy and pump vibration. The shape of the cut shows noticeable effect on the pump vibration and performance.
\end{abstract}

Index Terms-Flow induced vibration, double volute pump, different cut-shapes at blade exit, pump performance and vibration.

\section{INTRODUCTION}

The flow induced vibration in large and high power boiler feed pumps is one of the most challenging problems that limits the pump design and operating conditions. The pump design and pump requirements of flow rate and pressure delivery play a major role in inducing high pressure pulsation inside the pump. The blade exit shape and the effective gap between the rotor and stator are the most important design parameters in this regard. Pressure fluctuations inside the pump are mainly due to the interaction of the rotating impeller pressure field with the stationary circumferential pressure field generated by a vaned collection element, either volute tongue(s) or diffuser vanes. In centrifugal pumps, the impeller blades interfere with the diffuser vanes and produces pressure fluctuations downstream of the impeller.

Despite the long history of literature documenting forces on pump impeller, there are few detailed investigations into the effects of various impeller and volute combinations on these forces with a parallel examination of the hydraulic performance, Baun et al. [1]. The exact force magnitude and hydraulic performance depend on the exact geometrical details of pump design. When centrifugal pumps operate at partial

Manuscript received February 5, 2014; revised March 24, 2014. This work was supported by King Fahd University of Petroleum \& Minerals, project \# IN111050.

Atia E. Khalifa is with the Mechanical Engineering Department, King Fahd University of Petroleum \& Minerals, Dhahran 31261, Saudi Arabia (e-mail: akhalifa@kfupm.edu.sa). flow rates, the flow in the suction pipe starts to rotate with the impeller and finally a reverse flow from the impeller towards the suction occurs (inlet eye recirculation). Reverse flow causes some amount of energy losses at partial flow rates and the instability was attributed directly to the occurrence of the reverse flow and pre-rotation, and therefore the pump head becomes unstable; Breugehmans and Sen [2]. Improving the stability of the pump used to be at the expense of the pump performance and results in shifting the best efficiency point for the pump. The flow is not uniform around the impeller circumferentially, even at design point, due to the interaction between impeller and volute and the velocity profile at impeller exit; Guo and Maruta [3]. The pressure fluctuations depend on the flow rates and blade/vane angles. The largest blade pressure fluctuations occur at the trailing edge on the pressure side, but the magnitudes depend greatly on the number of vanes and vane angle. At off-design operating conditions, pressure fluctuations due to impeller-volute interaction occur mainly at blade passing frequency and its higher harmonics. The circumferential unevenness of fluctuations, which causes sidebands in frequency spectra, is dependent on the flow rate and guide vanes and become more significant at lower flow rates.

The largest vibration levels in large pump turbines are, in general, originated in the rotor stator interaction. This vibration has specific characteristics that can be clearly observed in the frequency domain, namely, harmonics of the moving blade passing frequency and a particular relationship among their amplitudes. The frequencies and their amplitudes depend on the pump design and the operating conditions. The amplitude of harmonics increased when certain conditions are achieved. The rise in the amplitudes is not equal for all harmonics; some are more affected than others. Rodriguez et al. [4] presented a theoretical analysis to predict and explain, in a qualitative way, such frequencies and amplitudes. The analysis incorporates number of blades, number of guide vanes, the RSI non-uniform fluid forces, and the sequence of interaction. The theoretical analysis is complemented with geometrical and timing considerations that allow going deep in the sequence on interaction rather than assuming excited diameter modes. The distance between the moving and stationary blades helps in determining the origin of the harmonics. Knowing the origin of these harmonics should guides to effective solutions in order to reduce their amplitudes. Many cases of impeller failures were caused by unusual radial gap between impeller and diffuser or volute tongue. Srivastav et al. [5] examined the effect of radial gap between impeller and diffuser on vibration and noise in a centrifugal pump under different flow conditions. It was shown that at maximum radial gap between the impeller and diffuser, the overall level of vibration and noise are minimum. 
Increasing the radial gap reduces vibration and noise levels, however, at the expense of pump performance in terms of head and efficiency. A clear correlation between vibration and noise is observed. If the flow rate is controlled by suction throttling, then the vibration level is higher than that corresponding to delivery throttling for small gaps in general, while this behavior may be reversed for large radial gaps. Makay et al. [6] studied the pressure pulsations arising from the interaction of the impeller and vaned diffuser in a $500 \mathrm{cfs}$ water supply pump, which caused numerous plant problems.

It is known that the performance of a centrifugal pump depends largely upon the flow patterns at the impeller exit to the extent that a small change in the edge form of the impeller blade causes a change in the pump performance. Koji et al. [7] examined the changes in the pump head and the flow pattern experimentally. They used pump impellers whose outlet angles are deformed stepwise by slicing off the blade on the suction or pressure sides. Slicing off the blade edge in the suction or pressure sides was found to increase the pump head due to the increase in the circumferential component in the absolute velocity. The blade outlet angle is one of the most important design parameters which can affect centrifugal pump performance. It controls the strength of the wake at the impeller exit. Shojaee and Boyaghchi [8] studied the performances of a centrifugal pump with different blade outlet angles both experimentally and numerically. The results show that when the outlet angle increases, the centrifugal pump performance handling viscous fluids improves. This improvement is due to decrease of wake at the exit of impeller.

The proposed work aims to experimentally test different cuts at the impeller blade exit in order to reduce the flow induced vibration of a boiler feed pump stage by increasing the effect clearance gap. The study correlates the blade exit shape with the effective gap under different operating conditions to select the best shape that produce minimum vibrations.

\section{EXPERIMENTAL SETUP}

Fig. 1 shows the experimental facility, pump, the instrumented test section, and sensors distribution around the impeller. Impeller diameter is $142 \mathrm{~mm}$ and the blade exit angle is 22.5 degree. The radial gap between the impeller and volute cutwater is $3.6 \mathrm{~mm}$ which is equivalent to $2.5 \%$ of the impeller diameter. Four identical impellers were manufactured; one impeller is kept without any cut in the blades' exits; and is used as a reference impeller. The second impeller is machined with a V-cut shape at the blade exit. The third impeller has a $\mathrm{C}$-cut shape at the blade exit. The fourth impeller has a straight cut at the blade exit. Fig. 2 shows the four impellers used in the present study. It should be mentioned here that the impeller shrouds remain un-touched with the original diameter for all impellers which means that the cutting is only in the blades between the upper and lower shrouds. Also it is important to mention that all the cuts of different shapes have the same cut area to produce the same effective increase in the clearance gap. Each impeller was dynamically balanced to minimize the effect of rotor imbalance as a source of vibration. A shaft-like extension was attached to the impellers to simulate the actual flow characteristics at the suction of the original boiler feed pump. The model pump speed is $3540 \mathrm{rpm}$ at $60 \mathrm{~Hz}$. Water at room temperature is used in all experiments.

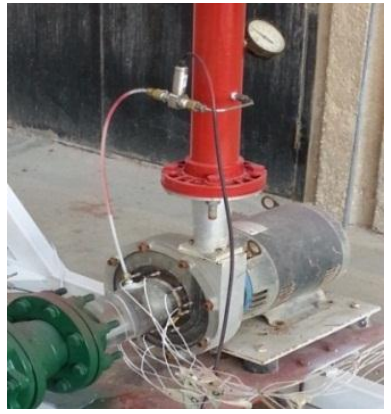

(a) Pump and instrumentation

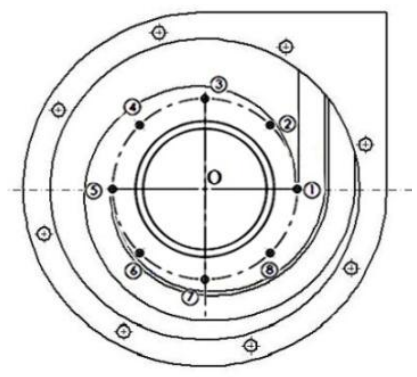

(b) Sensors distributed around impeller, uniform $45 \mathrm{deg}$. angles
Fig. 1. Pump assembly and instrumentation.

The flow rate is measured by a 4-inch electromagnetic flow meter (model Omega FMG3002-PP-D). The pressure at pump suction and discharge sides is measured using pressure transducers. The pressure fluctuations are measured around the impeller using a set of high-response dynamic pressure transducers (Omega DPX101-250) were placed flush within the volute Plexiglas cover plate to measure the unsteady pressure signals inside the pump. The output of all sensors and measured variables are connected to a data acquisition system (NI Compact DAQ 4-Slot USB Chassis with 4 Compact DAQ system modules NI 9234, each module has 4-Channel, $\pm 5 \mathrm{~V}$, $51.2 \mathrm{kS} / \mathrm{s}$ per Channel, 24-Bit). The dynamic pressure transducers are distributed every 45 degree around the impeller at blade exit as shown later in Fig. 6.

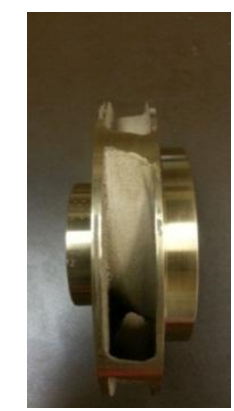

a. Impeller without blade cut

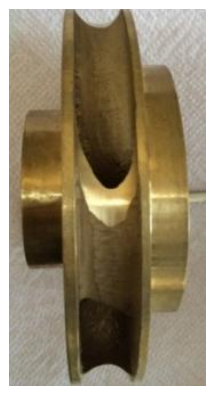

c. Impeller with C-cut

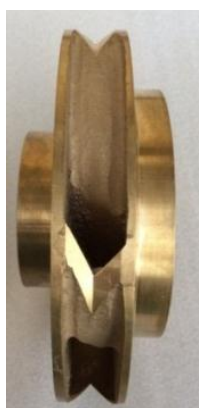

b. Impeller with V-cut

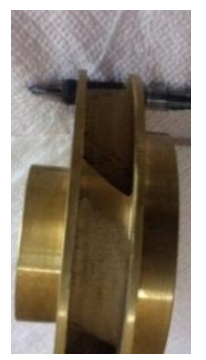

d. Impeller with straight cut (S-cut)
Fig. 2. The four impellers with different cuts at blade exit.

\section{RESULTS AND DISCUSSION}

The Pump total head is calculated as:

$$
H=\frac{p_{d}-p_{s}}{\gamma}+\frac{V_{d}^{2}-V_{s}^{2}}{2 g}+\Delta z
$$


where $p_{d} \& p_{s}$ are the static pressures at pump delivery and suction; respectively, $V_{d} \& V_{s}$ are the velocity at pump delivery and suction; respectively, and $\Delta z$ is the elevation between measuring locations of $p_{d} \& p_{s}$.

The Pump efficiency is calculated as:

$$
\eta=\frac{\gamma Q H}{\sqrt{3} * V * I * P F * \eta_{\text {motor }}}
$$

where $\gamma$ is the water specific weight in $\mathrm{N} / \mathrm{m}^{3}, Q$ is the volume flow rate in $\mathrm{m}^{3} / \mathrm{s}$, and $H$ is the pump head in $\mathrm{m}, V$ is the pump voltage in Volts, $I$ is the pump current in ampere, $P F$ is the motor power factor, and $\eta_{\text {motor }}$ is the motor efficiency. The measured pump performance curves; head, efficiency, and power, are shown in Fig. 3.

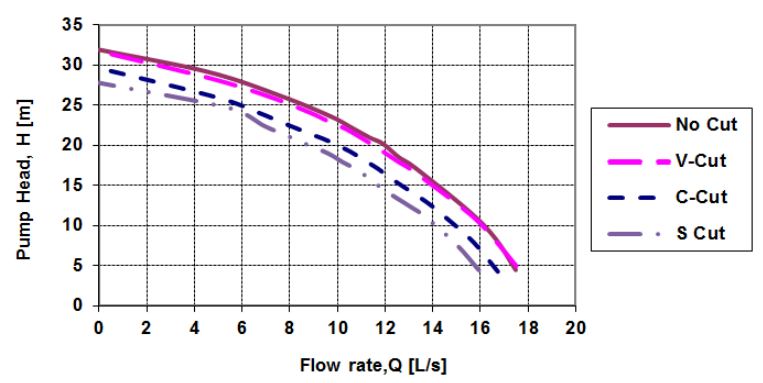

(a)

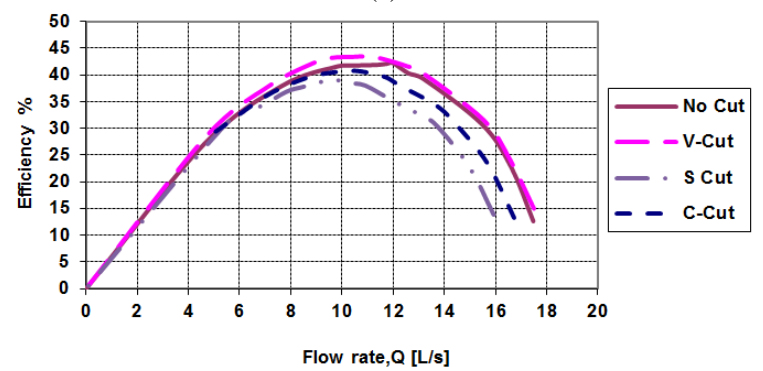

(b)

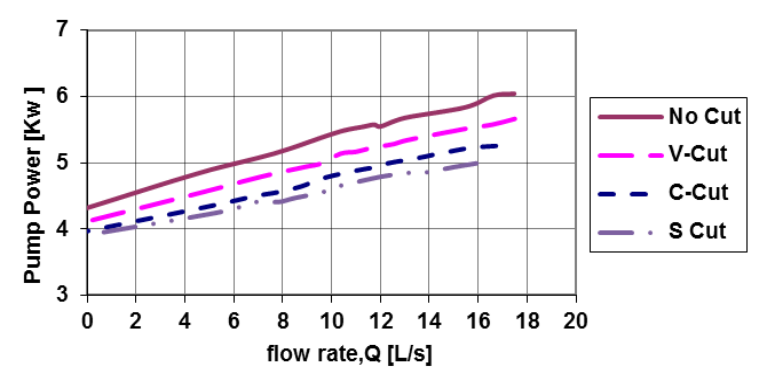

(c)

Fig. 3. Pump performance curves at different impeller blade exit cuts.

Compared to the original impeller with no Cut, one can see that all cuts produce less pump head due to the increase in the effective clearance gap. The head produced by the $\mathrm{V}$ - shaped cut impeller is very close to the original impeller. The V-cut reduces the head developed by the pump by about $5 \%$ at the best efficiency point. The existence of the V-cut reduces the power consumption by the pump which compensates for the head drop in calculating pump efficiency. In the other hand, a noticeable reduction in the pump performance is recorded with the impellers having C-cut and S-cut.

In order to study the flow induced vibration of this pump design, a set of dynamic pressure transducers are inserted flush through the volute cover plate to measure the pressure fluctuations around the impeller. Also, an accelerometer is installed on the pump volute casing to measure the pump vibration. Fig. 4 shows the time history of the pressure fluctuations as recorded by sensor number 2 associated with the spectrum analysis (FFT). The amplitude of pressure fluctuations are measured peak-to-peak. The full picture about pressure fluctuations is given by both the amplitudes and spectrum analysis, FFT. The spectrum dominant peak occurs at a frequency of $295 \mathrm{~Hz}$ which corresponds to $5 \mathrm{x}$ rpm or the first blade passing frequency (1st BPF or $5 \mathrm{x}$ ). Smaller peaks appeared at 10x rpm (2nd BPF) and 15x rpm (3rd BPF).

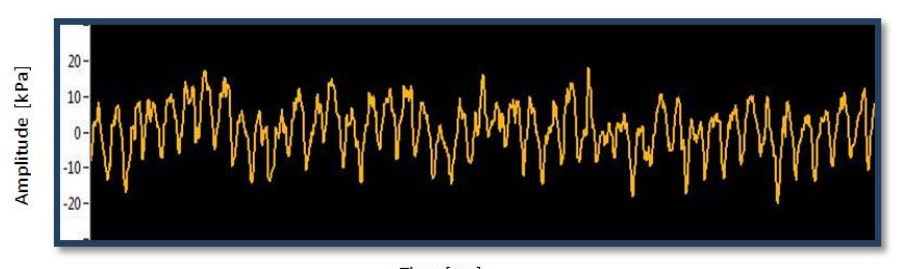

Time $[\mathrm{sec}]$

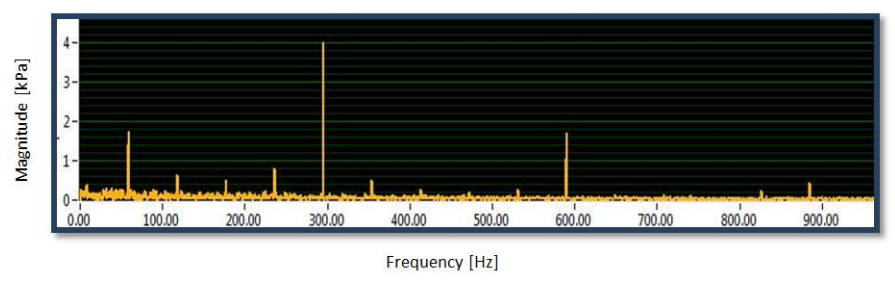

Fig. 4. Time history and frequency spectrum of pressure fluctuation signals inside the pump.

The measurements of the vibration of pump casing were carried out using B\&K 4507 Delta Tron accelerometers, with $100 \mathrm{mV} / \mathrm{g}$. The vibration occurs mainly at the first blade passing frequency $(5 \mathrm{x} \mathrm{rm})$; with smaller peaks appear at the second and third blade passing frequencies. This is in complete agreement with the measurements of internal pressure fluctuations of the model pump. The behavior of pump vibration is shown in Fig. 5. This behavior proves that the pump vibration is induced by pressure pulsations inside the pump due to the impeller-volute interaction. It also proves that measurements of the unsteady pressure field inside the pump can be used to identify and resolve the pump vibration problem.

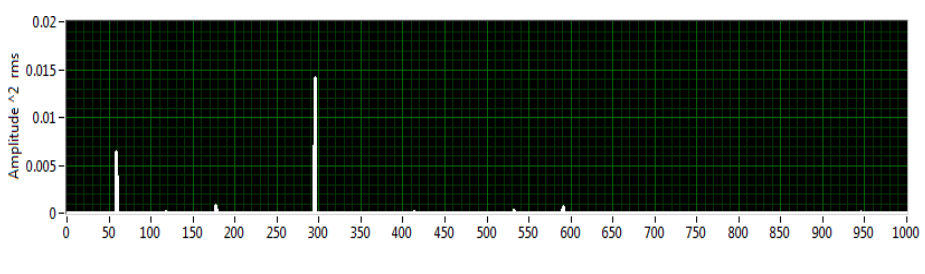

Frequency $[\mathrm{Hz}]$

Fig. 5. Frequency spectrum of pump casing vibration.

Fig. 6 shows the distribution of the amplitudes of pressure fluctuations around the impeller as recorded by the 8 dynamic pressure transducers. One can see that the highest peak to peak amplitudes are located near the interaction zones between the impeller blades and volute tongues (splitters).

The vibration of the pump casing under different flow ratios (ratio of the best efficiency flow rate) is presented in Fig. 7 for 
the four impellers. The minimum vibrations are measured at the design flow rate $(Q / Q n=1)$ in correspondence with the pressure fluctuation. The pump vibration is following the behavior of the amplitudes of pressure fluctuations inside the pump under variable flow rate conditions. Vibration increases as the pump operates at off-design conditions, both sides of the best efficiency operating point.

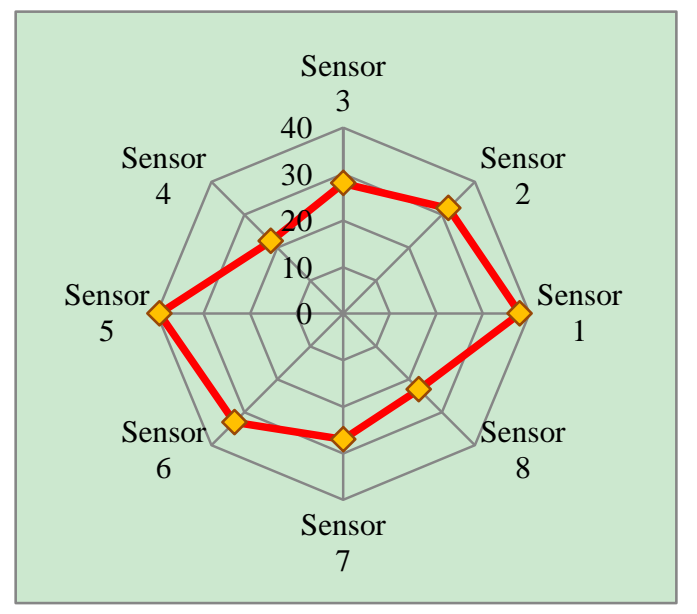

Fig. 6. Amplitudes of pressure fluctuation around the impeller.

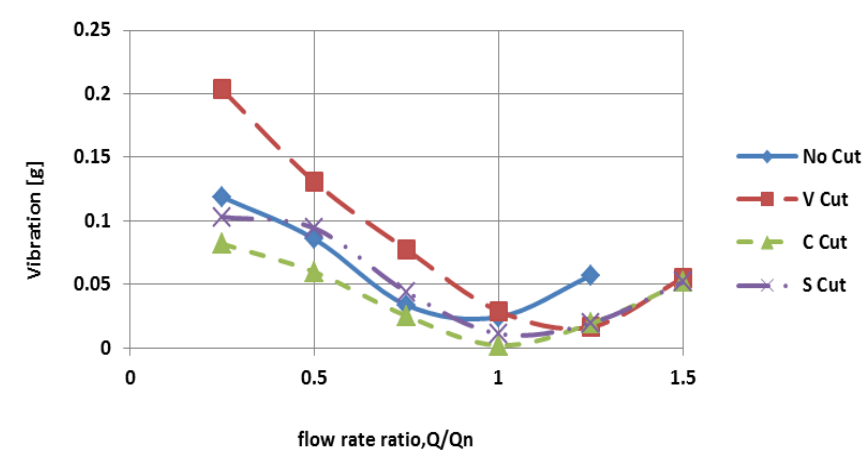

Fig. 7. Vibration behavior of the pump casing at different flow rates.

\section{CONCLUSIONS}

Experiments are carried out on a high pressure double volute centrifugal pump stage to study the effect of different cuts at the impeller blade exit on pump performance, pressure fluctuations inside the pump, and pump casing vibration. The impeller-volute interaction is an important design parameter and that design must be chosen carefully in each pump based on the pump design and operating conditions to minimize the flow induced vibration. The effective gap increased by cutting the impeller blade exit with different shapes. The impeller cuts reduced the amplitudes of pressure fluctuations inside the pump and consequently the pump vibration. The cuts are even more effective in reducing vibration at off-design flow rates. However, the reduction in vibration is achieved at the expense of the pump performance. A balance should be made between the pump performance and vibration based on the pump operation requirements.

\section{ACKNOWLEDGMENT}

The author thanks King Fahd University of Petroleum \& Minerals (KFUPM) for supporting this work under the funded project \# IN111050.

\section{REFERENCES}

[1] D. Baun and R. Flack, "Effect of volute design and number of impeller blades on lateral impeller forces and hydraulic performance," International Journal of Rotating Machinery, vol. 9, issue 2, pp. $145-152,2003$.

[2] F. Breugehmans and M. Sen, "Pre-rotation and fluid recirculation in the suction pipe of centrifugal pump," in Proc. 11th Int. Pump Sym., Texas A\&M Univ., 1982, pp. 165-180.

[3] S. J. Guo and Y. Maruta, "Experimental investigation on pressure fluctuations and vibration of the impeller in a centrifugal pump with vaned diffusers," JSME International Journal Series B, vol. 48, issue 1, pp. 136-143, 2005.

[4] C. Rodrigues, E. Egusquiza, and I. Santos, "Frequencies in the vibration induced by rotor stator interaction in a centrifugal pump turbine," Journal of Fluids Eng Trans ASME, vol. 129, issue 11, pp. 1428-1435, 2007.

[5] O. P. Srivastav, K. R. Pandu, and K. Gupta, "Effect of radial gap between impeller and diffuser on vibration and noise in a centrifugal pump," Journal of the Institution of Engineers (India): Mechanical Engineering Division, vol. 84, issue 1, pp. 36-39, 2003.

[6] E. Makay, P. Cooper, D. Sloteman, and R. Gibson, "Investigation of pressure pulsations arising from impeller/diffuser interaction in a large centrifugal pump," in Proc. Rotating Machinery Conference and Exposition, Somerset, NJ, 1993.

[7] K. Koji1, M. Mitsukiyo1, A. Eiji1, O. Isao, and L. Jinsheng, "Velocity distributions in the impeller passages of centrifugal pumps (Effects of outlet edge shape of the impeller blades on the pump performance)," Bulletin of JSME, vol. 28, no. 243, pp. 1963-1969, 1985.

[8] M. H. S. Fard and F. A. Boyaghchi, "Studies on the influence of various blade outlet Angles in centrifugal pump when handling viscous fluids," American J. Applied Science, vol. 4, issue 9, pp. 718-724, 2007.

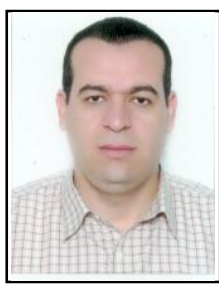

Atia E. Khalifa is an assistant professor of King Fahd Univ. of Petroleum \& Minerals, Mechanical Engineering Department, P.O. box 1068 Dhahran 31261, Saudi Arabia, he received his $\mathrm{PhD}$ degree in 2009. Dr. Khalifa's research areas include experimental and numerical fluid mechanics, turbomachinery, aerodynamics, combustion, and desalination. He is currently the director of pumping machinery lab at KFUPM. 